\section{Toothbrushing Frequency as It Relates to Plaque Development and Gingival Health*}

by

Niklaus P. Lang*

BOYD R. CUMMING***

HARALD LöE**

EPIDEMIOLOGICAL and experimental studies have demonstrated that gingival inflammation, as well as dental caries, is dependent upon the presence of bacterial plaque on the teeth. ${ }^{1-9}$ On a clean tooth surface, plaque begins to develop with the formation of a salivary- and glycoprotein containing pellicle ${ }^{10-13}$ into which bacteria may subsequently colonize.$^{14-18}$ Alterations in the bacterial flora, ${ }^{8,19}$ and changes in the chemical composition of plaque as it grows have been demonstrated. ${ }^{20-23}$ However, information on the rate of plaque growth and its pattern of development on the dentition is lacking.

It has been shown that if oral hygiene procedures are suspended, localized gingivitis develops in only 4-11 days and generalized gingivitis within 2-3 weeks. 8,19 On the other hand, resumption of oral hygiene procedures has resulted in a dramatic improvement in gingival health. ${ }^{8,19}$ Controversy exists, however, on how regularly bacterial plaque must be removed from the teeth in order to maintain gingival health. ${ }^{24-28}$ Epidemiological studies have indicated that less plaque occurs as the frequency of oral hygiene proedures increases. ${ }^{29-31}$ However, these studies have not considered the quality of toothbrushing and the patients' claims regarding the frequency of oral hygiene procedures performed. Another investigator has recently suggested that the gingiva might remain healthy, if plaque is removed completely only once every second day. ${ }^{28}$ How infrequently plaque may be removed without a deterioration in the health of the gingival tissues has not been established. Also, the effect of altered gingival health upon plaque formation has not been studied.

The purpose of the present study was to investigate the rate and pattern of plaque development in the den-

\footnotetext{
*Department of Periodontology, Royal Dental College, Aarhus, Denmark.

**Dental Research Institute, University of Michigan, Ann Arbor, Michigan.

***University of Saskatchewan, Saskatoon, Canada.
}

tition and to study the effect of gingival health upon plaque formation. Furthermore, it undertook to determine how frequently effective oral hygiene procedures must be performed to maintain gingival health.

\section{Materials AND MethodS}

Thirty-two dental students with clean teeth and healthy gingiva participated in the study. Since this study was to be carried out as a double blind test, the students were randomly assigned into four groups by a third person who did not participate in the experiment:

Group I removed plaque twice daily (every 12th hour).

Group II did so once every second day (every 48th hour).

Group III did so every third day (every 72 nd hour).

Group IV did so once every fourth day (every 96th hour).

The oral hygiene procedures were performed using Charters ${ }^{32}$ brushing technique supplemented by dental floss and interdental woodsticks for interdental cleansing. Using the Plak-Lite $\mathbb{R}$ disclosing system, ${ }^{33}$ a dental hygienist ensured that no plaque remained following the performance of oral hygiene.

In Group I, only one of the two daily brushings was supervised.

The amount of plaque was assessed by the same investigator for a period of 6 weeks according to the criteria of the Plaque Index System. ${ }^{34}$ The scoring was performed weekly, immediately before the scheduled cleansing so that the maximum plaque situation was assessed. At the same time, gingival health was assessed by a second investigator according to the criteria of the Gingival Index system. ${ }^{35}$ After the clinical assessment, the subjects rinsed with Plak-Lite $\AA$ disclosing solution and the anterior teeth were photographed under standardized conditions. From these photographs the surface area of fluorescent plaque was calculated planimetrically as described previously. ${ }^{33}$

The data from the clinical examinations were computerized and analyzed statistically.

\section{RESUlts}

The mean Plaque Index (Pl I) for all individuals within the different groups ranged from $0.17-0.27$ at the beginning of the experiment. Various amounts of plaque accumulation occurred in all groups during the experimental period (Figure 1). Group I, which accumulated plaque for 12 hours, obtained the least plaque (Pl I 0.44-0.81). In Group II, where plaque 


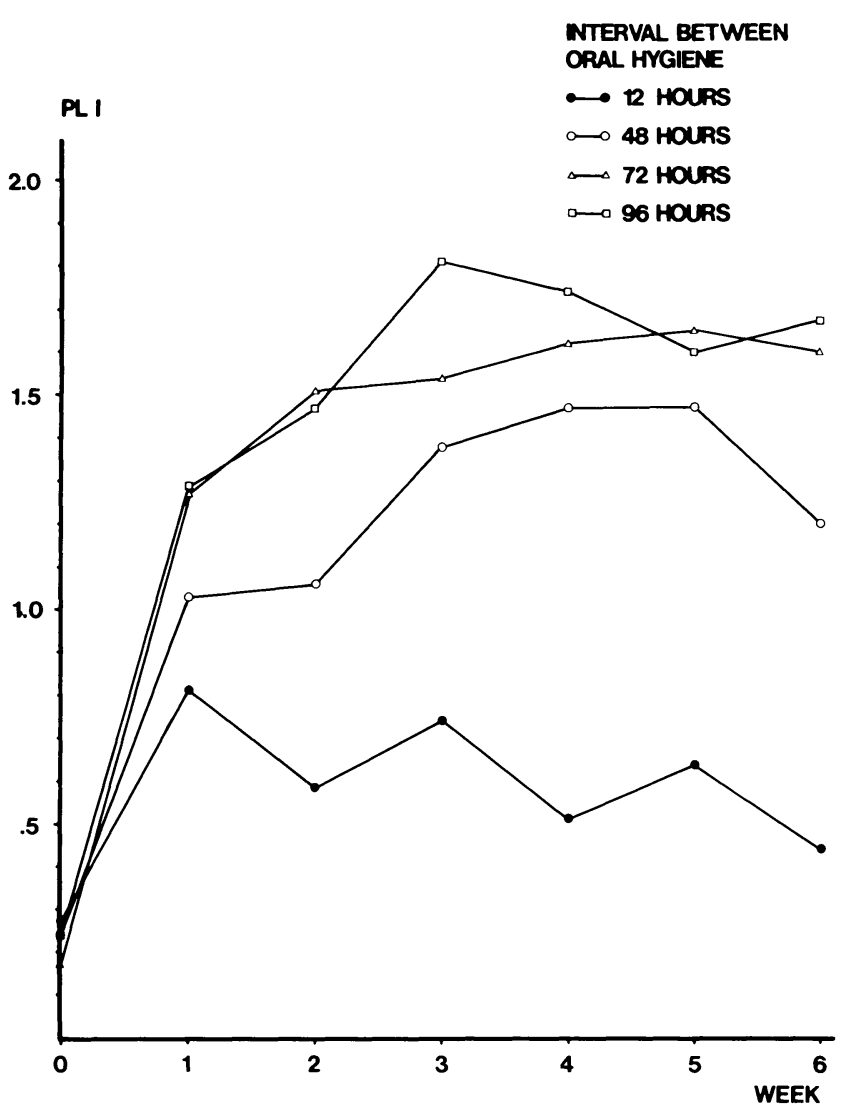

Figure 1. Mean Plaque Index for all tooth surfaces throughout the study of the individuals in the groups performing oral hygiene at various intervals. had accumulated for 48 hours, the amount of plaque tended to increase during the first 3 weeks (Pl I escalated from 1.03-1.38). After that time it leveled off and decreased during the 5th and 6 th week.

Groups III and IV, developing plaque for 72 and 96 hours respectively, formed similar amounts of plaque. Initially, the Pl I increased slightly and then leveled off after the 3rd week with a value of approximately 1.6. The percentage of surfaces with a Pl I score of 1 ranged from $0-100 \%$ among the individuals in Group I, while the tooth surfaces scoring $\mathrm{Pl} I=2$ or 3 ranged from $0-50 \%$ (Figure 2). The individuals accumulating plaque for 72 and 96 hours formed invisible plaque ( $\mathrm{Pl}$ $I=1)$ on 80 to $100 \%$ of their surfaces, while the individuals accumulating plaque for only 48 hours displayed greater variation $(50-100 \%)$ in the number of surfaces with plaque. The percentage of surfaces which consistently had visible plaque ( $\mathrm{Pl} \mathrm{I}=2$ or 3 ) varied among the individuals in Group II from 5-100\% and in Groups III and IV from 70-100\%.

Large differences were observed between the amounts of plaque formed in 12 hours (Figure 3 ). Individuals like No. 16 developed minimal amounts of invisible plaque, while other individuals, like No. 1, consistently displayed visible plaque. Differences between the amounts of plaque formed by the individuals within the other groups were not as obvious. Figure 3 shows

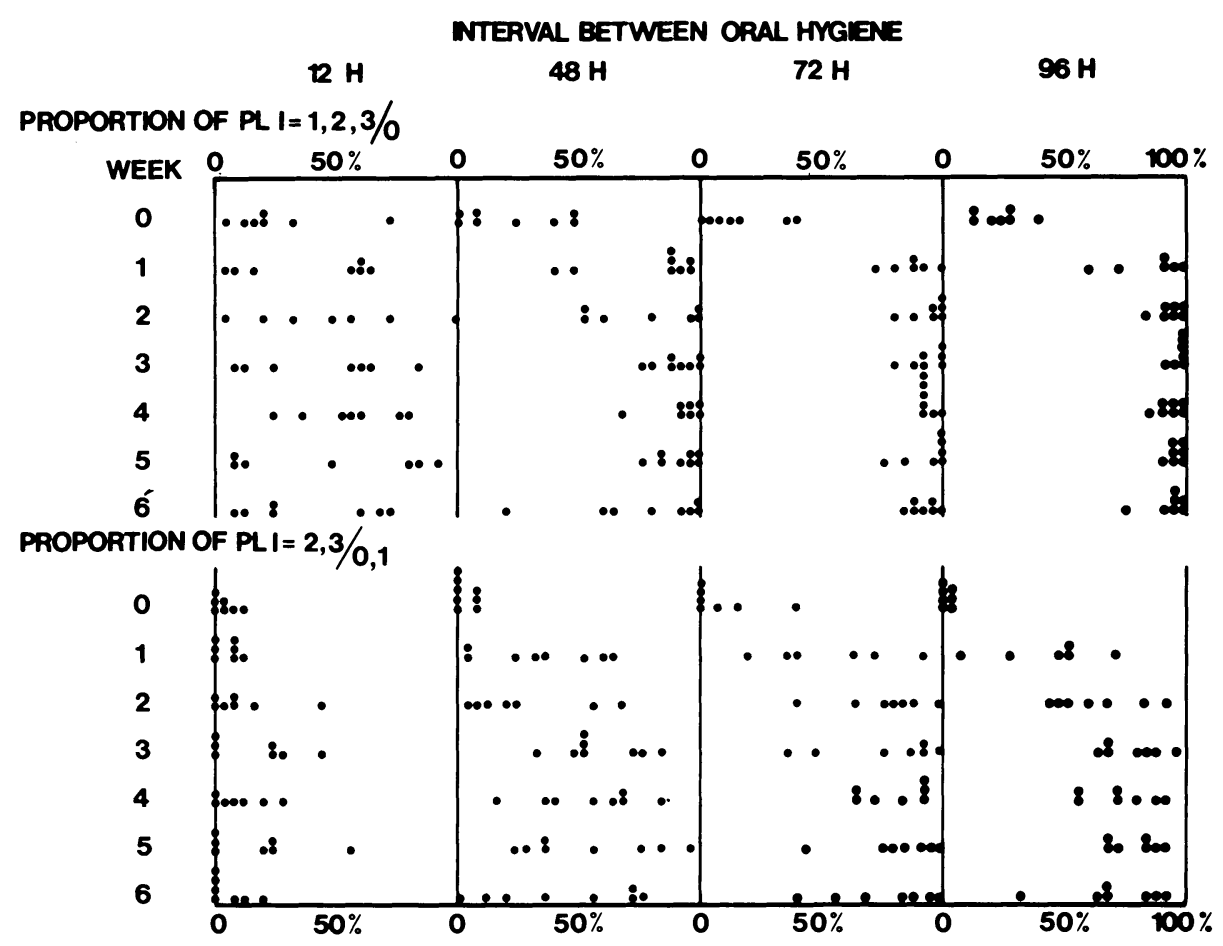

FIGURE 2. Distribution of the proportion of tooth surfaces scoring Plaque Index 1, 2 or 3 to 0 and Plaque Index, 2, 3 to 0 and 1 for each individual throughout the study. 


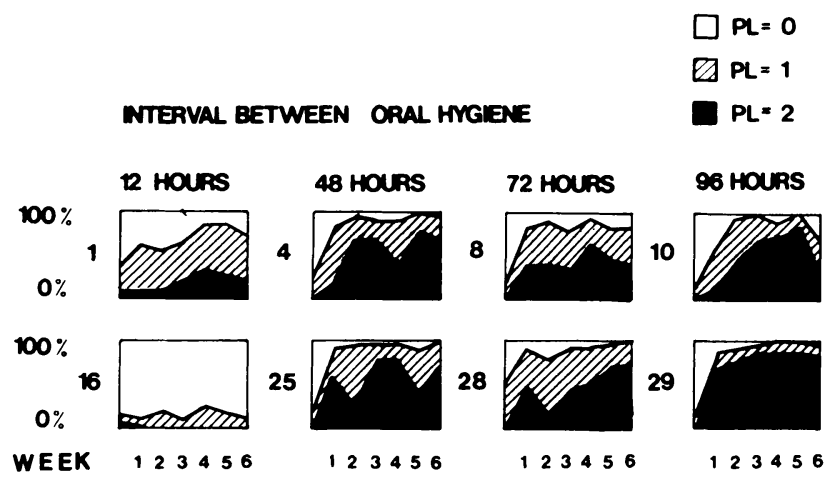

Figure 3. Mean Plaque Index on each examination for representative individuals from each group.

that the amount of plaque formed in nearly all the individuals varied from week to week. Minor fluctuations were observed in some individuals (e.g. No. 4, 8, 16, 29 ), whereas fairly large fluctuations were seen in others, such as No. 25, 28 and 10. However, all individuals in the study had plaque levels which were fairly characteristic for the duration of the period in which plaque was allowed to form.

The regularity with which Pl I scores of 0,1 or 2 and 3 occurred throughout the study on the facial, lingual and interproximal surfaces of each tooth in a representative individual (No. 11, 6, 5, 29) for each group is shown in Figure 4. A note of explanation concerning Figure 4: If a tooth surface on each examination throughout the study had the same Pl I score, either 1 or 2 and 3 , it is shown as $100 \%$. If, on the other hand, plaque was never observed on a particular surface, it is shown as $0 \%$. Consequently 0 and $100 \%$ represent the ultimate consistency, and values between these extremes indicated the proportion of times that plaque occurred. In Group I (represented by individual No. 11), plaque was rarely detected on the facial surfaces of any teeth except for the second molars which consistently had plaque. On the oral surfaces, only the molars displayed plaque on a regular basis. On the oral surfaces of the mandibular canines and premolars, plaque was detected on a proportion of the examinations. Some plaque was always present on the interproximals of the molars in Groups I; furthermore, these surfaces were scored $\mathrm{Pl}=2$ on a proportion of the examinations.

In the 48-hour group, some plaque was detected on nearly all surfaces. The distribution of Pl I scores of 2, however, was almost the same as the distribution of $\mathrm{Pl}$ I scores of 1 in Group I. The molars of the representative individuals for Groups II, III and IV had the highest percentage of $\mathrm{Pl} \mathrm{I}=2$ scores.

In the 96-hour group almost all surfaces except those

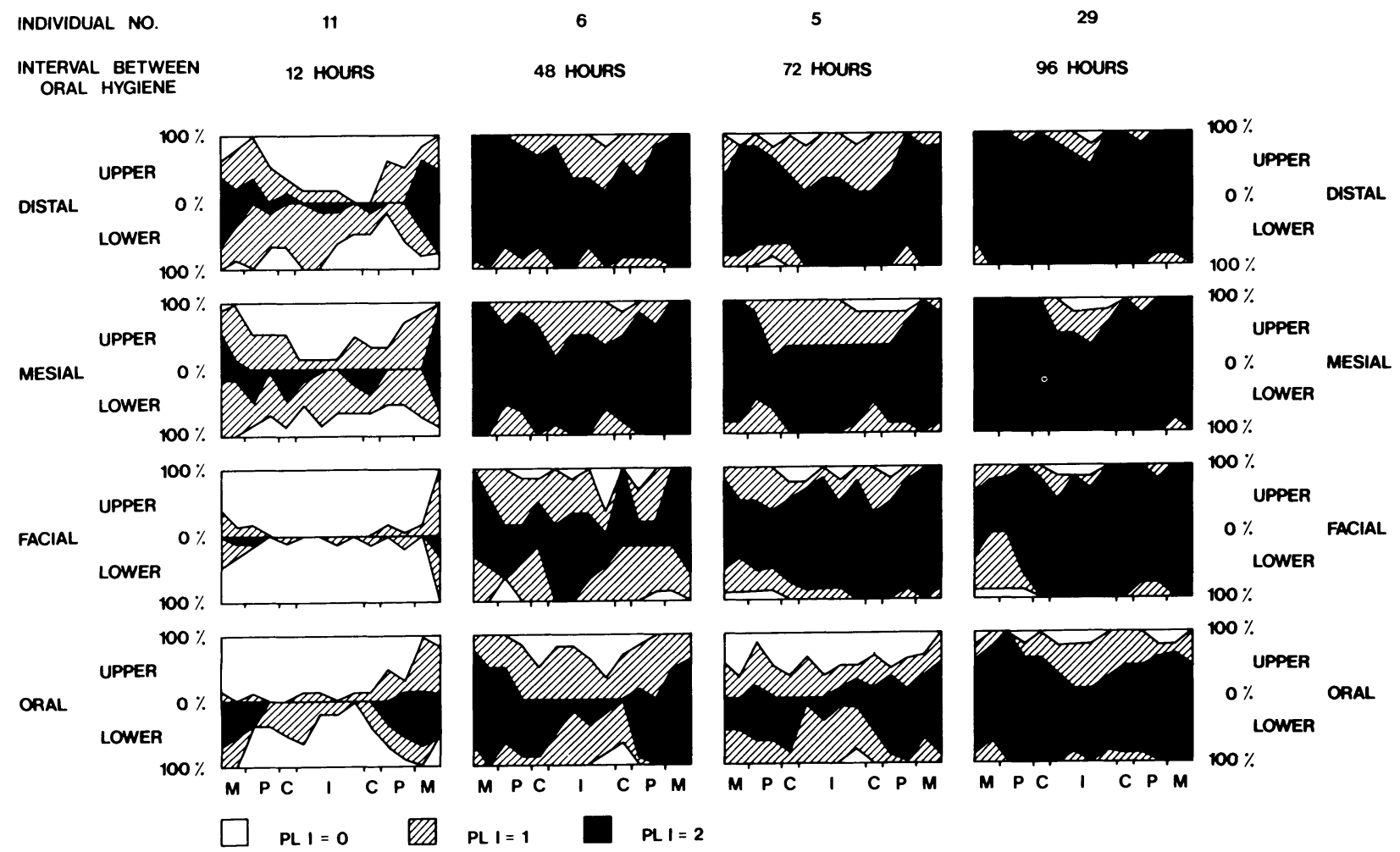

FIGURE 4. Consistency of the pattern of plaque distribution for representative individuals of each group (No. 11, $6,5,29)$. A tooth surface consistently having the same Plaque Index score (1, 2 or 3) throughout the study is shown as $100 \%$. A tooth surface upon which plaque was never detected is shown as $0 \%$. Values between these extremes indicate the proportion of times that plaque occurred. M: Molars, P: Premolars, C: Canines, I: Incisors. 
of the maxillary incisors displayed visible plaque. In general, the greatest differences in the amount of visible plaque in Groups II, III and IV were observed on the facial and oral surfaces, while the interproximals almost consistently were covered with visible plaque. The pattern of plaque distribution for each individual in the study was unique. For example, among the many peculiarities of the pattern showed by individual No. 11 was the observation that the oral surface of the lower left lateral incisor was always clean, whereas the lower right lateral incisor was normally involved with plaque. Similar peculiarities could be seen in the other individuals represented (e.g. No. 6, 5, 29).

The planimetric measurements of the surface area of the anterior teeth covered with fluorescent plaque showed characteristic amounts of plaque for the four groups (Figure 5). However, in Groups II, III and IV a slight increase in the surface covered by plaque was noted at the beginning of the experimental period, and a slight decrease was observed at the end of the period.

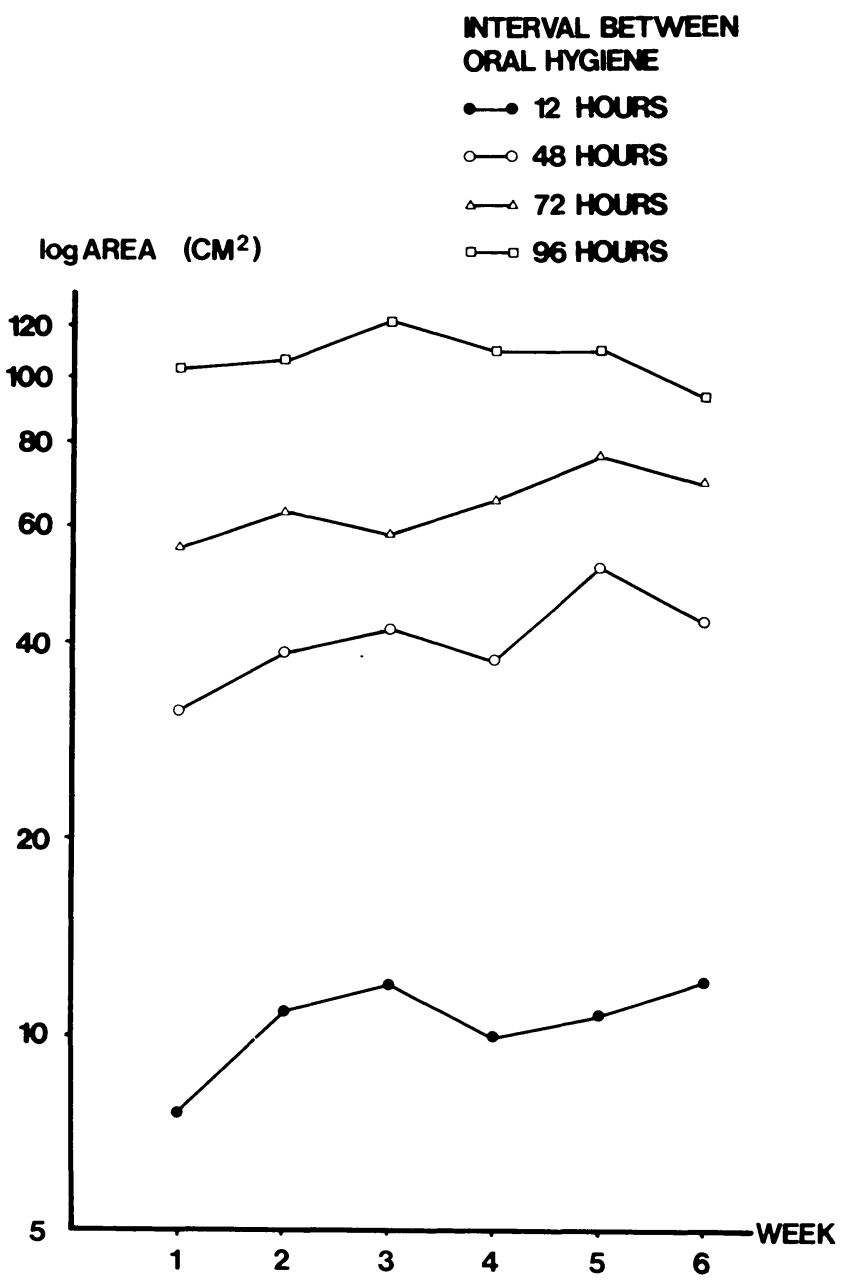

Figure 5. The log mean area of fluorescent plaque on the interproximal and labial surfaces of the anterior teeth for each group.
The mean Pl I of these same anterior teeth as well as the posterior teeth confirmed these measurements (Figure 6). In Group IV some individuals formed quite different amounts of plaque on the anteriors from one week to another, as shown by both surface area measurements and PI I assessments (Figures 7a,b). The occlusal extension of plaque, as measured by the area of fluorescence, and its thickness at the gingival margin, as measured by the Pl I, correlated almost linearly in the range $\mathrm{Pl} \mathrm{I}=0.4-1.8$ (Figure 8 ).

Only Groups III and IV developed gingivitis during the experimental period (Figure 9). The mean Gingival Index (GI), which ranged from $0.08-0.11$ for all groups at the start of the study, remained on the same level (GI $=0.08-0.25$ ) in Groups I and II.

The mean GI of the group performing oral hygiene every 72 nd hour increased after the second week from 0.16 to 0.27 . A second increase from 0.30 to 0.44 was observed after the fifth week. In Group IV, performing oral hygiene at an interval of 96 hours, the mean GI increased almost linearly from 0.13 the first week to 0.87 at the end of the experiment.

In each single examination the variation between the individuals within the different groups was small (Fig-

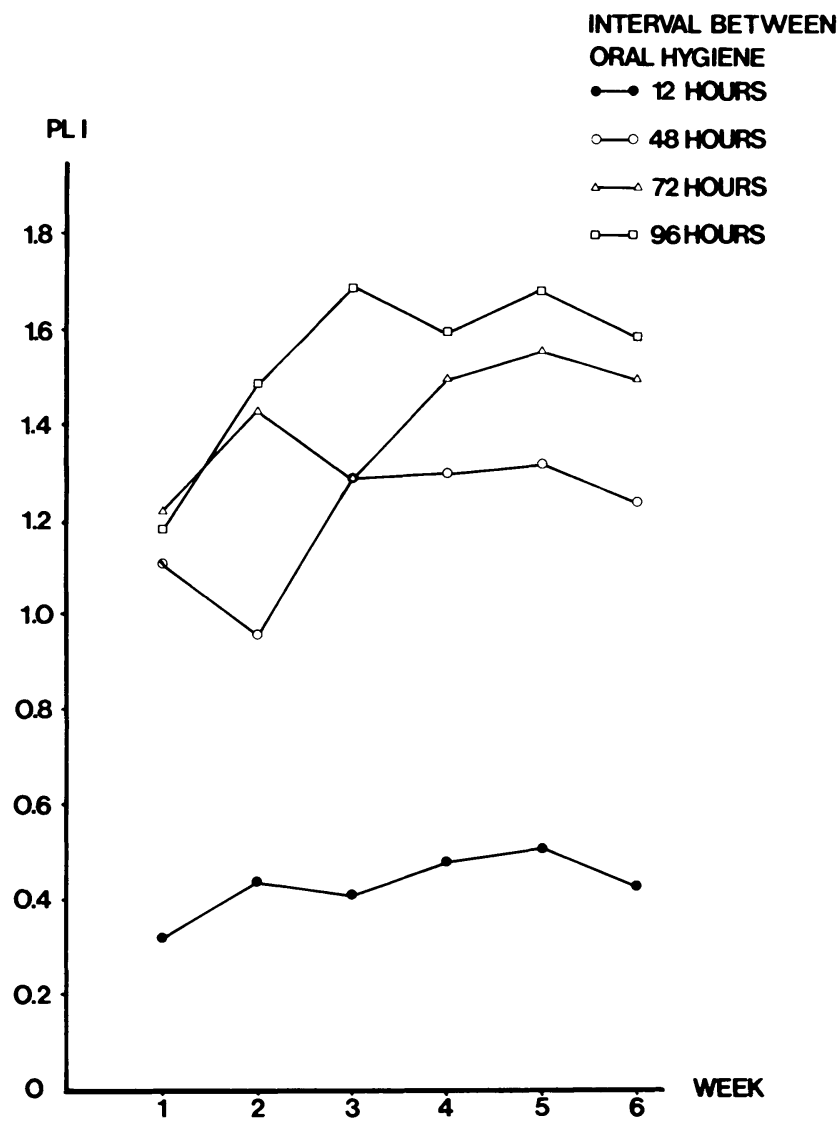

FIgURE 6. Mean Plaque Index for the interproximal and labial surfaces of the anterior teeth for each group. 


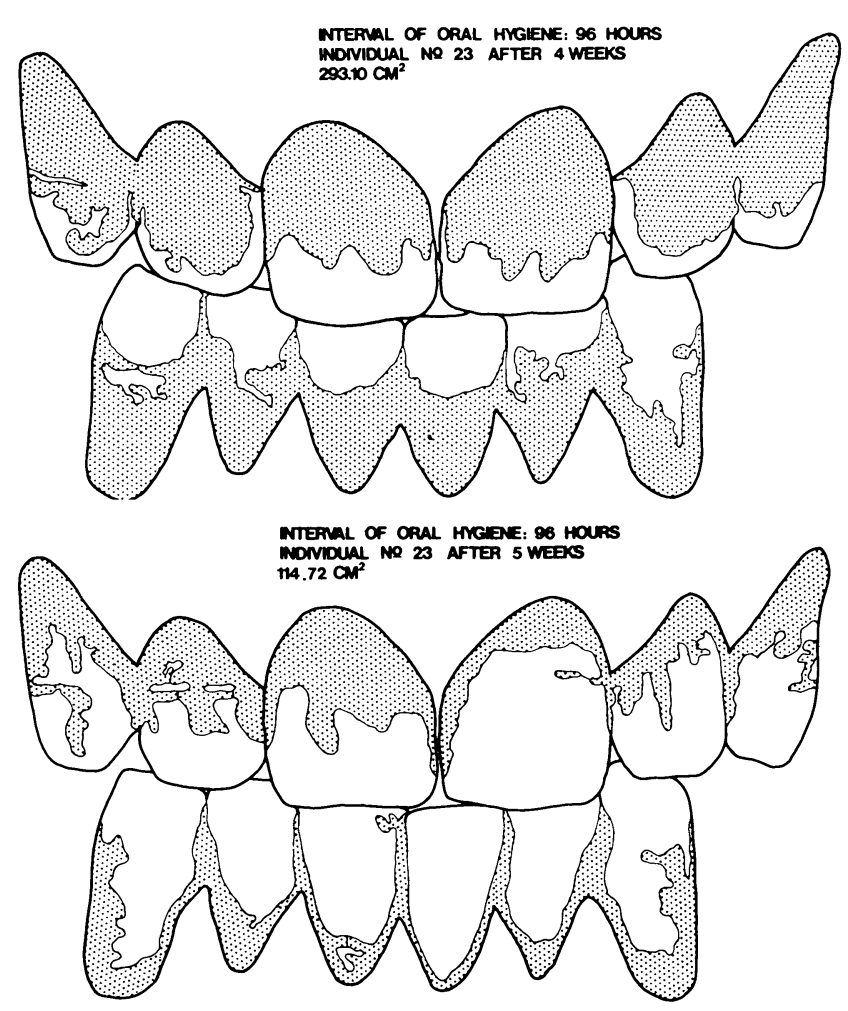

FigurE 7. Tracing of the area of fluorescent plaque on the anterior teeth of one individual in Group IV (No. 23): $A$. at the 4 th week examination; $B$. at the 5 th week examination.

ure 10). The increase of the mean GI was due to an increased number of GI scores of 1 in all groups and examinations, except for the final scoring in Group IV (Figure 10). At the beginning, no GI scores of 2 were given while about $10 \%$ of all surfaces in all individuals scored $\mathrm{GI}=1$. This percentage remained consistent

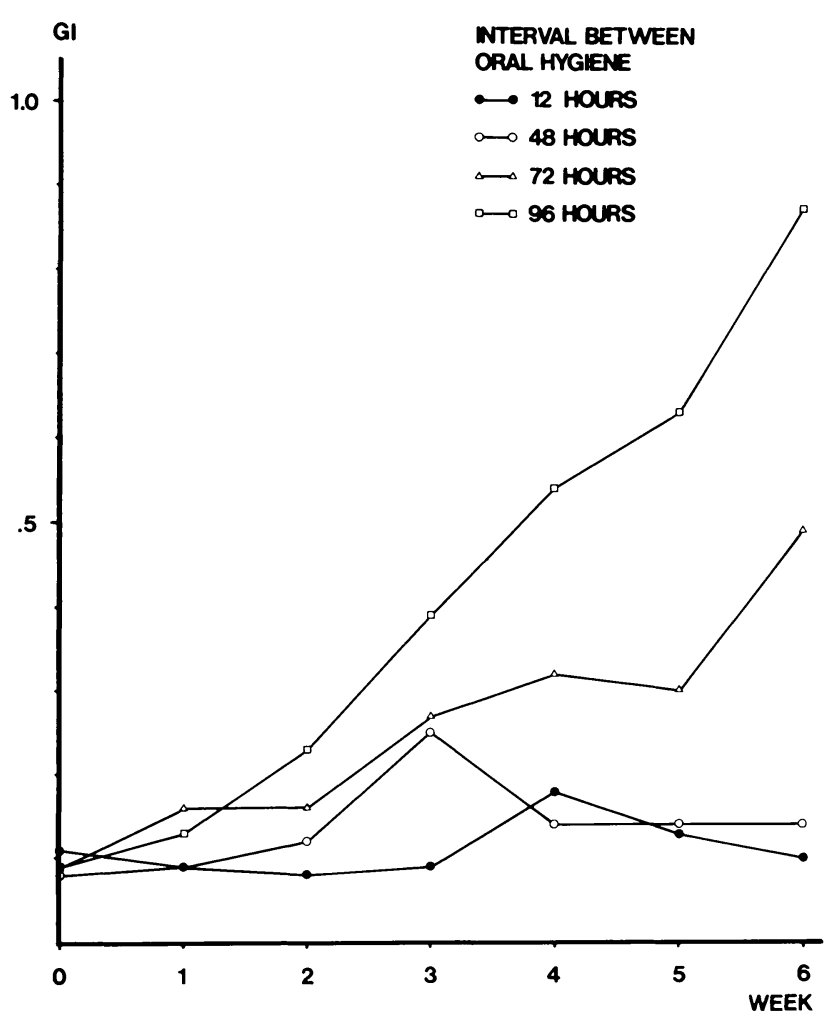

FIGURE 9. Mean Gingival Index throughout the study of the individuals in the groups performing oral hygiene at various intervals.

throughout the experiment in Groups I and II (Figure 10). However, occasional variations in scoring occurred in both these two groups.

In Group III the percentage of GI scores 1 and 2 increased slightly during the experimental period and showed greater variation. In Group IV about $75 \%$ of

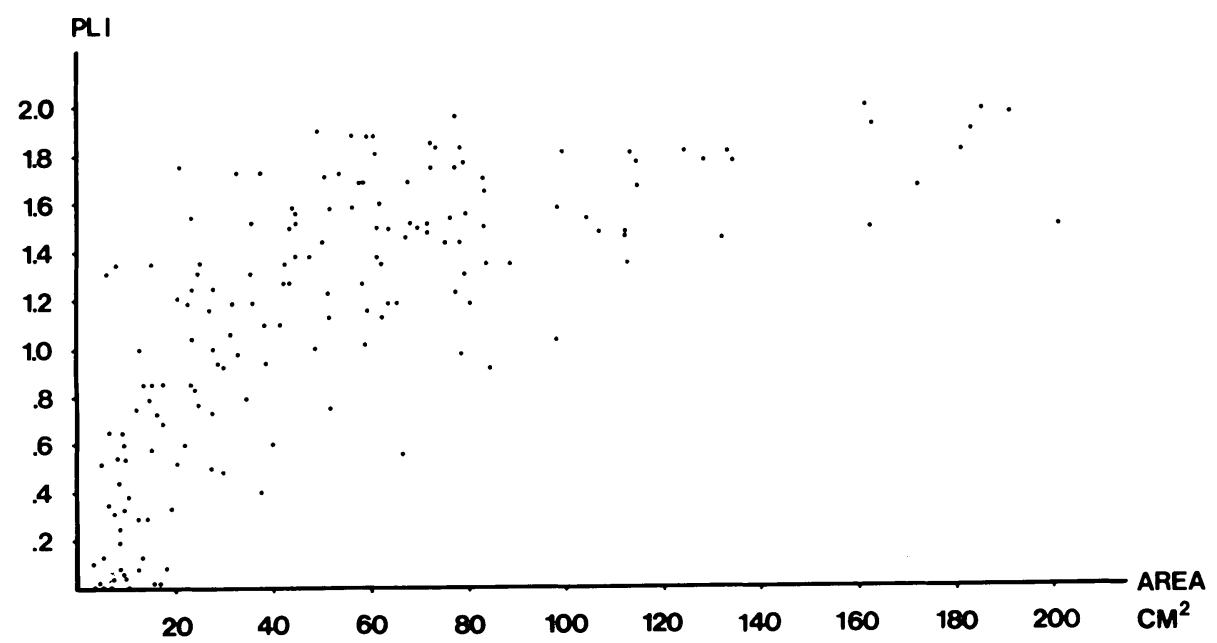

FIGURE 8. Correlation between Plaque Index scores and the mean area of fluorescent plaque (anterior teeth). 


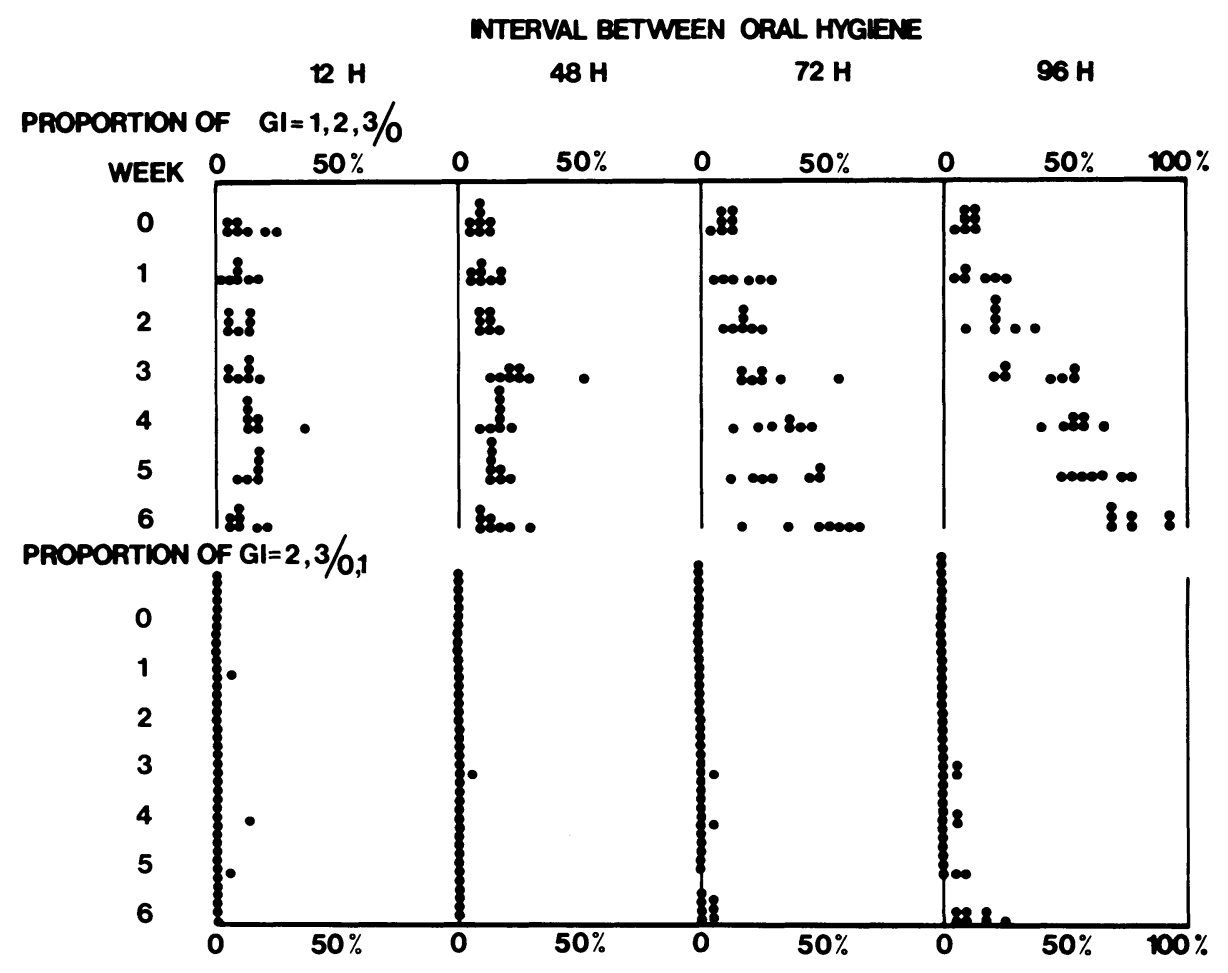

FIGURE 10. Distribution of the proportion of gingival units scoring Gingival Index 1, 2 or 3 to 0 and Gingival Index 2, 3 to 0 and 1 for each individual throughout the study.

all surfaces scored $\mathrm{GI}=1$ and 2 at the end of the experiment. In addition approximately $10 \%$ of all surfaces scored GI $=2$ on this examination. The increase in the percentage of GI scores 1 and 2 in Group IV was remarkably constant $(10-15 \%)$, whereas in Group III the increase varied more between the individuals (Figure 11). Some individuals (e.g. No. 8) showed almost the same increase as some individuals in Group IV (e.g. No. 10) and others (e.g. No. 28) were similar to individuals in Group II (e.g. No. 4).

At the end of the experiment, the patterns of gingivitis incidence in Groups I and II were about the same (Figure 12). GI scores of 1 appeared mainly in the buccal gingiva of the maxilla and the mandible adjacent to the canines and first premolars and in the interproximal gingiva of the molars. In Group III, mild inflammation $(\mathrm{GI}=1)$ mainly occurred in the interproximal and facial gingiva of the lower premolars and molars and in the oral gingiva of the lower anteriors and molars. In the maxilla, GI scores of 1 were given to the gingiva adjacent to most of the premolar and molar areas. Moderate inflammation $(\mathrm{GI}=2)$ was localized in the facial gingiva of the canines and first premolars. The gingivitis profile of Group IV revealed mild gingivitis in nearly all the facial and interproximal gingivae of canines, premolars and molars. The oral aspect of the maxilla showed the least inflammation. Moderate inflammation was primarily localized in gingiva adjacent to premolars and molars. The development of gin-

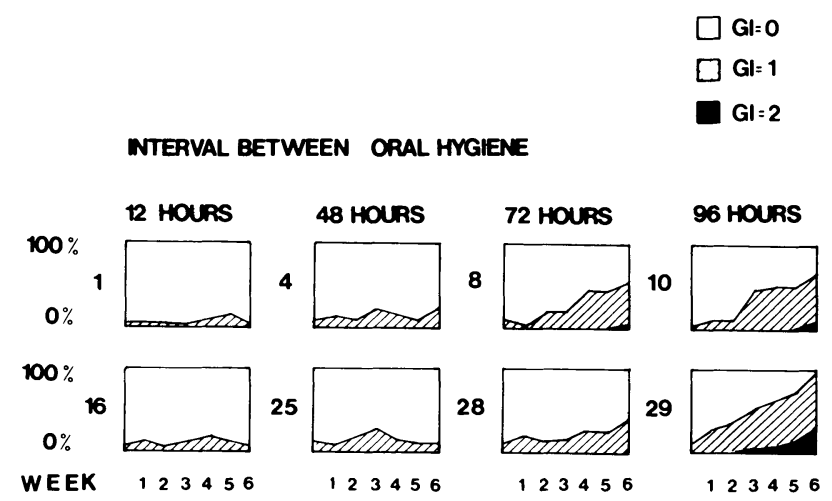

FIGURE 11. Mean Gingival Index at each examination for representative individuals from each group.

givitis by surfaces was studied in Group IV (Figure 13). It showed nearly the same pattern as demonstrated in Figure 12. Mild gingivitis started in the facial gingiva of the maxilla and the oral gingiva of the lower anteriors after 2 weeks. After 4 weeks the molar gingiva also showed mild inflammation in some areas. The oral gingiva of the maxilla remained healthy up to the fifth week. However, at the end of the experiment, it showed quite a remarkable number of areas scoring $\mathrm{GI}=2$ (moderate inflammation).

\section{DISCUSSION}

The present study has demonstrated that the amount of plaque which an individual accumulated during a 


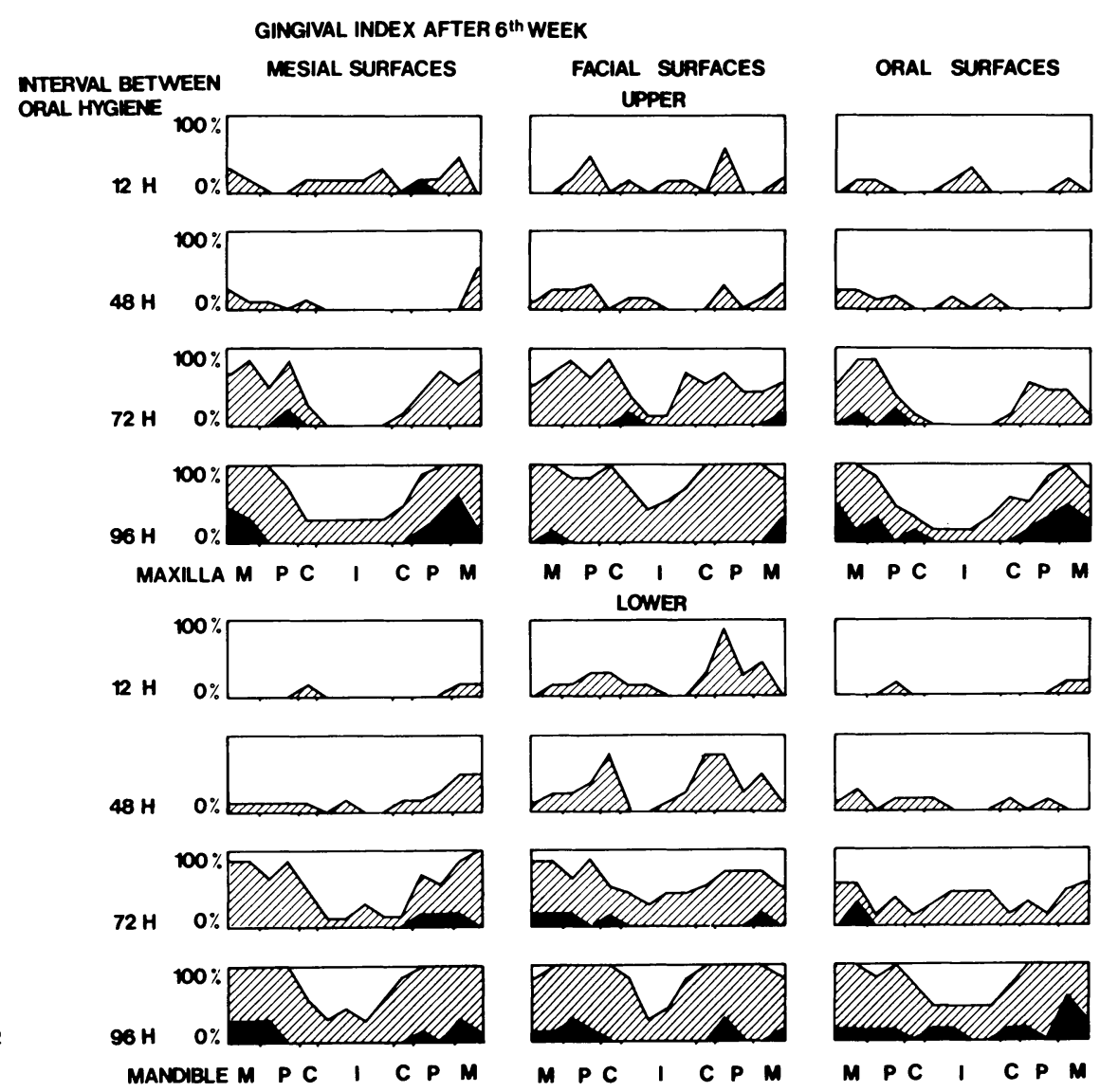

FIGURE 12. Mean distribution of gingivitis on the mesial, facial and oral gingiva for each group on the final examination. M: Molars, P: Premolars, C: Canines, I: Incisors.

given period is fairly consistent. However, occasional variations in the rate of plaque formation were found within the individuals of all the groups.

Generally, the rate of plaque formation increased during the first 3 weeks and reached a level which was characteristic for each group as gingivitis scores became higher. This phenomenon was revealed by both the $\mathrm{Pl}$ I assessments and the measurements of the area fluorescence of the anterior teeth. The factors which affected the rate of plaque formation were not identified in the present study. However, it has been shown that the rate of gingival exudation increases before the clinical ap-

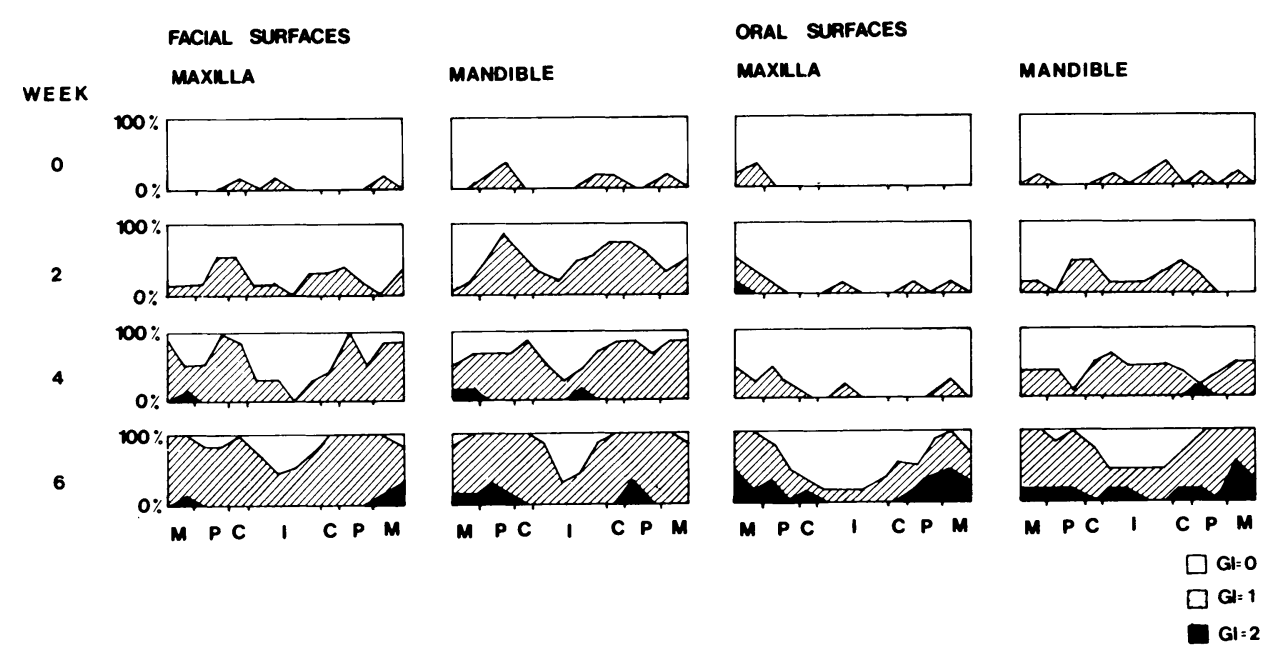

FIGURE 13. The pattern of development of gingivitis in individuals performing oral hygiene at an interval of 96 hours. M: Molars, P: Premolars, C: Canines, I: Incisors. 
pearance of gingivitis, ${ }^{36}$ and these alterations in gingival exudation may have contributed to the amount of plaque formed. Furthermore, while the plaque was completely removed from the teeth, the number and composition of the residual bacterial flora in the saliva may have changed and influenced the rate of plaque accumulation.

Plaque formation began in the interproximal areas of the premolars and molars after which it started to develop on the interproximal surfaces of the anteriors and on the facial surfaces of the premolars and molars. The oral surfaces accumulated the least plaque. This pattern did not always correlate with the pattern of gingivitis development. Gingival inflammation generally started in the interproximal areas of the premolars and molars and in the facial gingiva of the premolars and canines, and the anterior interproximal and the facial and oral molar areas subsequently developed gingivitis. Finally, gingivitis occurred in the facial and oral anterior gingiva. The observation that the facial gingivae of the canines and first premolars were inflamed at a very early stage although they were usually plaque free may be explained by the fact that some individuals in the study had a very narrow width of keratinized gingiva, an observation which has been described previously. ${ }^{37,38}$ Whether or not these areas have a lower local resistance and response upon irritation has been discussed. ${ }^{38}$

The present study has also shown that the performance of effective oral hygiene procedures at intervals of 48 hours is compatible with the maintenance of gingival health. This is in agreement with previous reports. ${ }^{28}$ However, if the interval between complete removal of bacterial plaque exceeded 48 hours, gingivitis developed.

Since only minor differences in the amount of plaque between the 48, 72 and 96 hour groups were revealed by the Pl I system, it appears that factors other than the amount of plaque determine its pathogenicity. Bacterial studies have indicated that it takes in excess of 48 hours for the plaque to become predominantly gramnegative. ${ }^{8,19}$ The appearance of gram-negative cocci and rods may be one reason for the increase in the pathogenicity of dental plaque. On the other hand, chemical changes in plaque as it matures ${ }^{20}$ may be of significance. Furthermore, it is reasonable to assume that a longer exposure of the gingival tissues to plaque results in greater irritation of these structures.

Variations in the prevalence of gingivitis in the group accumulating plaque for 72 hours may be due to differences in host tissue resistance. In addition, the time taken for the bacterial flora to change varies between individuals. ${ }^{19}$ Therefore it may be that variations in the degree of gingivitis in Group III resulted from differences in susceptibility of the individuals as well as in the nature of the plaque present.
In all cases in the present study, surfaces scoring $\mathrm{GI}=2$ were preceded in one or more of the previous examinations by $\mathrm{GI}=1$. Furthermore, in individuals where some surfaces were scored $\mathrm{GI}=2$, nearly $100 \%$ of the remaining surfaces were scored $\mathrm{GI}=1$. This indicates that the first change observed in inflamed gingiva is the changing colour of the tissues. These findings disagree with a recently published report ${ }^{39}$ that sulcus bleeding upon probing is the first clinical sign of gingivitis.

Although all tooth surfaces were completely plaquefree after each oral hygiene procedure, a consistent pattern of plaque development was evident, starting in the interproximal areas of the premolars and molars. Since these surfaces are the same ones which consistently remain dirty in the average patient without special home care instruction, ${ }^{40}$ it is obvious that special attention has to be paid to interdental cleansing when motivating patients. Furthermore, it is apparent from this study that complete removal of plaque only once every second day is more valuable from a prevention point of view than performing numerous inadequate or ineffective oral hygiene procedures each day.

Measurements of the area of fluorescence on the anterior teeth correlated nearly linearly with the mean Pl I of the same teeth in the range of $0.4-1.8$. These findings are in agreement with those of an earlier study. ${ }^{33}$ They also corresponded with the mean Pl I for all tooth surfaces. Although plaque accumulates in the anteriors at a slower rate, these teeth can probably be used as representative for the mouth when assessing the oral hygiene situation in epidemiological studies. In clinical practice, however, prevention of periodontal disease requires careful full mouth assessments, since plaque accumulates on the posterior teeth first.

\section{SUMMARY}

Thirty-two dental students with excellent oral hygiene and clinically healthy gingiva were randomly assigned into 4 groups by a third person who did not participate in the experiment:

Group I removed plaque every 12 th hour,

Group II did so once every 48th hour,

Group III did so once every 72nd hour and

Group IV did so once every 96th hour.

Using the Plak-Lite $\AA$ disclosing system, a dental hygienist ensured that no plaque remained after the performance of oral hygiene procedures.

The amount of plaque was assessed for a period of 6 weeks according to the criteria of the Plaque Index system (Pl I), immediately prior to the scheduled cleans- 
ing. At the same time, gingival health was assessed according to the criteria of the Gingival Index system (GI). In addition, the area of fluorescent plaque after rinsing with Plak-Lite $\AA$ solution on the anterior teeth was calculated planimetrically.

The following observations were made:

1) The rate of plaque accumulation increased before the appearance of clinically detectable gingivitis and levelled off as gingivitis developed. It was apparent that alterations in gingival exudation and other clinical changes of the tissues affected the rate of plaque formation.

2) Plaque occurred first in the interproximal areas of the premolars and molars and then started to form on the interproximal surfaces of the anteriors and the facial surfaces of the premolars and molars. The oral surfaces accumulated the least plaque.

3) An almost linear correlation between the thickness of plaque at the gingival margin, as measured by the PI I, and its occlusal extension, as measured by the area of fluorescent plaque, was found. However, with minute amounts of plaque ( $\mathrm{Pl} \mathrm{I}<0.4$ ) the Pl I was more sensitive.

4) Anterior teeth may be representative for the mouth when assessing oral hygiene in epidemiological studies. In clinical practice, however, prevention of periodontal disease requires careful full mouth assessments.

5) Effective oral hygiene procedures at intervals of 48 hours are compatible with gingival health. However, if the intervals between complete removal of bacterial plaque exceeded 48 hours, gingivitis developed.

6) Since only minor differences in the amount of plaque between the 48-, 72-, and 96-hour groups were revealed by the Pl I system, it appears that other factors than the amount of plaque determine its pathogenicity.

\section{REFERENCES}

1. L $\varnothing \mathbf{v d a l}$, A., A. Arno and I. Waerhaug: Incidence of clinical manifestations of periodontal disease in light of oral hygiene and calculus formation. J. Am. Dent. Assoc., $56: 21,1958$.

2. Schei, O., J. Waerhaug, A. Løvdal and A. Arno: Alveolar bone loss as related to oral hygiene and age. J. Periodontol., 30:7, 1959.

3. Greene, J. C.: Periodontal disease in India: Report of an epidemiologic study. J. Dent. Res., 39:302, 1960.

4. Greene, J. C.: Oral hygiene and periodontal disease. Dent. Abstr., 8:764, 1963.

5. Löe, H.: Epidemiology of periodontal disease. An evaluation of the relative significance of the etiological factors in the light of the recent epidemiological research. Odontol. T., 71:479, 1963.

6. Russel, A. L.: Geographical distribution and epide- miology of periodontal disease. J. Indoes. Dent. Ass. 13:19, 1963.

7. Russel, A. L.: Epidemiological research. 1960-1963. J. Am. Dent. Assoc., 68:820, 1964.

8. Löe, H., E. Theilade and S. Børglum Jensen: Experimental gingivitis in man. J. Periodontol., 36:177, 1965.

9. Ramfjord, S. P., R. D. Emslie, J. C. Greene, A. J. Held and J. Waerhaug: Epidemiological studies of periodontal diseases. Am. J. Public Health, 58:1713, 1968.

10. Leach, S. A., P. Critchley, A. B. Kolendo and C. A. Saxton: Salivary glycoproteins as components of the enamel integuments. Caries Res., 1:104, 1967.

11. Hay, D. I.: The absorption of salivary proteins by hydroxyapatite and enamel. Arch. Oral Biol., 12:937, 1967.

12. Mayhall, C. W.: Concerning the composition and source of the acquired enamel pellicle of human teeth. Arch. Oral Biol., 15:1327, 1970.

13. S $\varnothing$ nju, T. and G. Rølla: Chemical analysis of a salivary glycoprotein with bloodgroup substance-and virus inhibition activities. Acta Pathol. Microbiol. Scand. 79:95, 1971.

14. Meckel, A. H.: The formation and properties of organic films on teeth. Arch. Oral Biol., 10:585, 1965.

15. Gibbons, R. J. and S. B. Banghort: Synthesis of extracellular dextran by cariogenic bacteria and its presence in human dental plaque. Arch. Oral Biol., 12:11, 1967.

16. Gibbons, R. J. and R. J. Fitzgerald: Dextran-induced agglutination of Streptococcus mutans and its potential role in the formation of microbial dental plaque. J. Bact. 98:341, 1969.

17. v. Houte, J., C. E. de More and H. M. Jansen: Synthesis of iodophilic polysaccharide by human oral streptococci. Arch. Oral Biol. 15:263, 1970.

18. Rølla, G.: Adsorption of dextran to saliva-treated hydroxyapatite. Arch. Oral Biol. 16:527, 1971.

19. Theilade, E., W. H. Wright, S. Børglum Jensen and $\mathrm{H}$. Löe: Experimental gingivitis in man II. A longitudinal, clinical and bacteriological investigation. J. Periodont. Res., $1: 1,1966$.

20. Kleinberg, I. and G. N. Jenkins: The pH of dental plaque in the different areas of the mouth before and after meals and their relationship to the $\mathrm{pH}$ and rate of flow of resting saliva. Arch. Oral Biol., 9:493, 1964.

21. Ritz, H. L.: Microbial population shifts in developing human dental plaque. Arch. Oral Biol., 12:1561, 1967.

22. Kleinberg, I. and G. Hall: $\mathrm{pH}$ and depth of gingival crevices in different areas of the mouths of fasting humans. J. Periodont. Res., 3:109, 1968.

23. Graf, H.: The glycolytic activity of plaque and its relation to hard tissues pathology-recent findings from intraoral pH telemetry research. Int. Dent. J., 20:426, 1970 .

24. Kitchin, P. C.: The role of home care in dental caries control. In: Muhler, J. C. and Hine, M. K. A symposium on preventive dentistry (eds.), pp. 117-121. C. V. Mosby Co., St. Louis, 1956.

25. Ariaudo, A. A.: How frequently must patients carry out effective oral hygiene procedures in order to maintain gingival health? J. Periodontol., 42:309, 1971.

26. Greene, J. C.: How frequently must patients carry out effective oral hygiene procedures in order to maintain gingival health? J. Periodontol., 42:310, 1971.

27. Arnim, S. S.: How frequently must patients carry out effective oral hygiene procedures in order to maintain gingival health? J. Periodont. 42:311, 1971. 
28. Löe, H.: How frequently must patients carry out effective oral hygiene procedures in order to maintain gingival health? J. Periodontol., 42:312, 1971.

29. Greene, J. C. and J. R. Vermillion: Oral hygiene index: a method of an epidemiological study. J. Dent. Res. 39:302, 1960.

30. Alexander, A. G.: The effect of frequency of brushing and the type of bristle used on gingival inflammation, plaque and calculus accumulation. Dent. Pract. Dent. Rec., 20:347, 1970.

31. McKendrick, A. J. W., L. M. H. Barbenel and W. D. McHugh: The influence of time of examination, eating, smoking and frequency of brushing on the oral debris index. J. Periodont. Res. 5:205, 1970.

32. Charters, W. J.: Ideal tooth brushing. J. Dent. Res. 4:XI, 1922.

33. Lang, N. P., E. Østergaard and H. Löe: A fluorescent plaque disclosing agent. J. Periodont. Res., 7:59, 1972.

34. Silness, J. and H. Löe: Periodontal disease in preg- nancy II. Correlation between oral hygiene and periodontal condition. Acta Odontol. Scand., 22:121, 1964.

35. Löe, H. ad J. Silness: Periodontal disease in pregnancy I. Prevalance and severity. Acta Odontol. Scand. 21:533, 1963.

36. Löe, H. and P. Holm-Pedersen: Absence and presence of gingival fluid in normal and inflamed gingivae. Periodontics 3:171, 1965.

37. Ainamo, J. and H. Löe. 1966. Anatomical characteristics of gingiva. A clinical and microscopic study of the free and attached gingiva. J. Periodontol., 37:5, 1966.

38. Lang, N. P. and H. Löe: The relationship between the width of keratinized gingiva and gingival health. J. Periodontol., 43:623, 1972.

39. Mühlemann, H. R. and S. Son: Gingival sulcus bleeding-a leading symptom in initial gingivitis. Helv. Odontol. Acta, 15:107, 1971.

40. Cumming, B. R. and H. Löe: Consistency of plaque distribution in individuals without special homecare instruction. J. Periodont. Res. 8:94, 1973.

\section{Announcement}

\section{AMERICAN BOARD OF PERIODONTOLOGY}

The following changes in procedure have been adopted by the Board:

1. Approved applications are now valid for six years, instead of five years.

2. Candidates have the option until December 1, 1974 of submitting three case reports under the new rules or five reports under former regulations. The deadline for this option had previously been announced as December 1, 1973.

Written Examination

The next written examination of the American Board of Periodontology will be given on October 23, 1973 in San Antonio, Texas. Deadline for application is September 1. For further information contact the Executive Secretary, Dr. Robert L. Reeves, University of Southern California School of Dentistry, 925 W. 34th Street, Los Angeles, California 90007.

\section{Clinical-Oral Examination}

The Clinical-Oral Examination of the American Board of Periodontology was conducted at Indiana University School of Dentistry on April 2-4, 1973. The following candidates, having passed the examination, have now been certified as Diplomates:

Herbert Abrams

Hugh H. Bruner, Jr.

Howard Buckwald

Philip S. Caplan

Elliott H. Dickler

David Dolgin

Harley A. Ellinger, Jr.

Michael E. Fritz

Louis H. Green

Patrick C. Haggerty
Milton Klempert

Dan M. Loughlin

Bernard F. Podlin

Joseph E. Rowan

Thomas J. Schafer

Miles H. Scheffer

Alvin J. Snyder

Richard Tavtigian

Richard C. Weiss

Richard H. Yamada 